\title{
Statistical Evaluation of Semi-Analytical, Analytical, and Numerical Models of the Serial Production Lines
}

\author{
Viktor Ložar*, Tihomir Opetuk, Hrvoje Cajner, Neven Hadžić, Jerolim Andrić
}

\begin{abstract}
Production lines are the backbone of the manufacturing industry. To gain the best profit out of a line it is necessary to design each line using the production system engineering. Therefore, three approaches can be used, the numerical, the analytical, and the semi-analytical approach. The aggregation method, finite state method, and the numerical approach are statistically compared concerning the analytical approach using the STATISTICA software. We analyzed the interaction between the input data and the output data for the finite state method in an illustrative example, using a full factorial design and the Design Expert software.
\end{abstract}

Keywords: analytical approach; Design Expert; production lines; production system engineering; STATISTICA

\section{INTRODUCTION}

The production industry has a big influence on the economic growth of a country, [1]. One job in the production industry generates five to seven jobs in the rest of the economy, [2]. To increase such benefits, it is important to take care of the production facilities, for example, the serial production lines, which are the backbone of the manufacturing industry.

The serial production line consists of machines and buffers which are placed in a line. Such lines can be short or long, depending on the product type. The amount of the required products determines the level of automatization. The combination of the machines and buffer capacities influences productivity. For this reason, designing such lines is getting more and more attention.

Therefore, two different approaches can be used, the heuristic approach and the systematic approach which is known as the production system engineering (PSE). The heuristic approach is based on trial-and-error experiments, which generate unpredictable results. The systematic approach was developed in the past five decades mainly in the automobile industry. The PSE is suitable for designing new production lines or improving existing ones, [3]. With such an approach the designer can predict the costs, the energy consumption, [4], or evaluate the impact of production on the environment, [5]. The PSE determines the key parameters of a production line, which can be used to identify bottlenecks or to achieve a leaner production line reducing the buffer capacities without losing the target performance, [6].

To calculate the key parameters the following three models can be used: the numerical, the semi-analytical and the analytical approach.

\section{BRIEF LITERATURE REVIEW}

In the Year 1962 Sevest yanov published the analytical solution for a two-machine single buffer line in the steadystate response, [7] called the serial Bernoulli production line. For longer serial lines with more machines and buffers, this approach was not suitable except for a three-machine line under specific circumstances, [8].

To calculate longer production lines, semi-analytical methods were introduced, the decomposition method, the aggregation method, and the finite state method. The decomposition method was published 1986, [9]. Since then the algorithm was enhanced twice to gain higher efficiency, [10] and to be closer to the real production lines, [11]. The aggregation method was published 1990 based on an iterative solution, [12]. The aggregation method uses a series of forward and backward aggregations of the whole line into a single machine, until the convergence of results. The finite state method was developed recently based on the analytical approach valid for an arbitrary number of machines and buffers, [13]. This new analytical approach is based on the constitutive matrixes, which generate the transition matrix for the whole line. This approach is exact but very timeconsuming and therefore the finite state method was developed, [14].

The numerical approach is a common way today but needs 1-3 months of preparations before some valuable results can be generated, [15]. Therefore, the designers must be well skilled and trained in the usage of the simulation software.

The aggregation method, finite state method, and numerical approach were never statistically compared by the analytical approach. Therefore, the data from the supplement of the paper [16] and the software STATISTICA will be used in this work to fill the gap. To get an overview of the interaction between the input data and the output data a design of experiment approach with the software Design Expert is used on an illustrative example.

\section{THE KEY PARAMETERS}

Here we provide a summary of the key parameters. More details can be found in the paper [16].

Bernoulli serial line consists of machines, buffers and conveyors which can be shown as circles, rectangles and arrows, Fig. 1. 


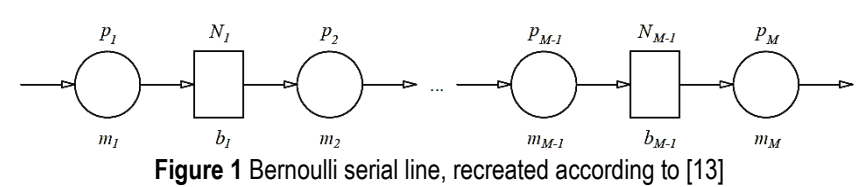

Every approach calculates the key parameters production rate $P R$, the work-in-process, WIP, the probability of blockage, $B L$, and the probability of starvation, $S T$.

The production rate of the whole line, $P R$, is the intersection between the probability that the last machine is up and the probability that the previous buffer is not empty, therefore it is expressed as:

$P R=P\left[\left\{\right.\right.$ machine $m_{M}$ is up $\} \cap\left\{\right.$ buffer $N_{M-1}$ is not empty $\left.\}\right] .(1)$

The work-in-process, $W I P_{i}$, describes the average number of semi-products in each buffer and can be defined with following expression:

$W I P_{i}=\sum_{h_{1}=0}^{N_{1}} \sum_{h_{2}=0}^{N_{2}} \cdots \sum_{h_{M-1}=0}^{N_{M-1}} h_{i} P_{h_{1} h_{2} h_{3} \cdots h_{M-1}}$,

where $h_{i}$ express the number of semi-products and $P_{h_{1} h_{2} h_{3} \cdots h_{M-1}}$ the steady-state probability of the system. For the whole line, the work-in-process can be calculated as follows:

$W I P=\sum_{i=1}^{M-1} W I P_{i}$

The probability of blockage for the penultimate machine, $B L_{M-1}$, is expressed by the following intersection of probabilities:

$$
B L_{M-1}=P\left[\left\{m_{M-1} \text { is up }\right\} \cap\left\{b_{M-1} \text { is full }\right\} \cap\left\{m_{M} \text { is down }\right\}\right] .
$$

The probability of blockage for the previous machines, $B L_{i}$, can be defined with the following expression:

$$
\begin{aligned}
& B L_{i}=P\left[\left\{m_{i} \text { is up }\right\} \cap\left\{b_{i} \text { is full }\right\} \cap\left\{m_{i+1} \text { is down }\right\} \cup\right. \\
& \left.\cup\left\{m_{i} \text { is up }\right\} \cap\left\{b_{i} \text { is full }\right\} \cap\left\{b_{i+1} \text { is blocked }\right\}\right] .
\end{aligned}
$$

The probability of starvation of the $i^{\text {th }}$ machine is the intersection between the probability that the buffer $i-1$ is empty and that the probability of the machine $i$ is up, therefore it is expressed as:

$$
S T_{i}=P\left[\left\{b_{i-1} \text { is empty }\right\} \cap\left\{m_{i} \text { is up }\right\}\right], i=2,3, \ldots, M .
$$

\section{THE STATISTICAL COMPARISON}

The main goal of this paper is to compare the different approaches and methods by using the test dependent sample approach. Therefore, the software tool STATISTICA will be used to compare the key parameters $\left(P R, W I P_{i}, W I P, B L_{i}\right.$, and $S T_{i}$ ) of the aggregation method, finite state method, numerical approach with the key parameters of the analytical approach. Such comparison will be done for 12 lines in 4 cases with 3, 4, 5 and 6 machines. The data generated in [16] will be used. Longer production lines with more than 6 machines in a line are not considered because the CPU demand for the analytical approach is too high to get results in a reasonable time.

The 12 production lines for each case are getting out of 200 randomly generated production lines. To sample out the 12 lines, the performance measures by the analytical approach for each case will be the base for the application of the following criteria's, [16]:

Line 1: line with the smallest $P R$

Line 2: line with the largest $P R$

Line 3: line with the $P R$ in between production rates of lines 1 and 2

Line 4: line with the smallest $W I P$

Line 5: line with the largest $W I P$

Line 6: line with the WIP in between the work-in-process of lines 4 and 5

Line 7: line with the smallest $B L_{M-1}$

Line 8: line with the largest $B L_{M-1}$

Line 9: line with the $B L_{M-1}$ in between the probability of blockage of lines 7 and 8

Line 10:line with the smallest $S T_{M}$

Line 11:line with the largest $S T_{M}$

Line 12:line with the $S T_{M}$ in between the probability of starvation of lines 10 and 11.

\subsection{The Results}

The statistical test (the differences in mean between dependent samples) approach using significance level of $1 \%$ to compare the key parameters of the aggregation method, the finite state method, the numerical approach with the analytical approach. The comparison of the key parameters in the case of a 3-machine line shows no significant differences according to the analytical approach. Except for the $W I P_{2}$ of the numerical approach is higher than the $W I P_{2}$ of the analytical approach. This deviation is still less than $5 \%$. The comparison of the key parameters in the case of a 4machine line shows no deviations for all key parameters. The comparison of the key parameters in the case of a 5-machine line shows no deviations in the major key parameters, except the following:

- The $P R$ of the numerical approach is higher than the $P R$ of the analytical approach.

- The $B L_{1}$ of the aggregation method shows significantly lower values than the $B L_{1}$ of the analytical approach.

- The $B L_{1}$ of the numerical approach shows significantly lower values than the $B L_{1}$ of the analytical approach.

- The $B L_{2}$ of the numerical approach shows significantly lower values than the $B L_{2}$ of the analytical approach.

- The $S T_{5}$ of the numerical approach shows significantly lower values than the $S T_{5}$ of the analytical approach. 
The comparation of the key parameters in the case of a 6-machine line shows no deviations of all parameters except the $B L_{1}$ and $S T_{6}$. These parameters are in the numerical approach less than in the analytical approach but still in an error range of $5 \%$.

In general, all methods generate the same results as the analytical approach, except the numerical approach for some parameters of longer lines. In such cases, the numerical approach generates smaller values for some parameters as the $B L$ and the $S T$.

\section{THE DESIGN OF EXPERIMENTS}

To design an experiment there can be used a wide spectrum of techniques. The Trial and Error approach is maybe the oldest technique to solve experiments. This technique is not searching for the best solution to a problem, it is search for just a solution. Such a technique is useless when it is not allowed to make errors in the experiment. This Technique can be applied in a wide range, from mathematical to sociology experiments, [17].

The one factor at the time (OFAT) technique is not the best practice to analyses experiments but because of its simplicity, it is still in use. One factor is changing while the rest is fixed, if the measured output is better than before the next experiment will use this changed value and change another factor. Comparing to the factorial design, more runs are needed. Further, OFAT is not taking care of the interactions of factors or about the optimal settings of factors.

The $2^{k}$ full factorial design technique takes $k$ factors at two levels, a high level, and a low level into consideration. This means if there are $k=3$ factors, there will be $2 \times 2 \times 2$ $=8$ experiments needed to discover all possible combinations. The goal of this technique is to discover the effect of each factor and their interactions on the response variable of the experiment. If the number of factors increases the number of experiments will exponentially increase, which makes the method suitable for up to 4 or 5 factors. Expectations can be done in computational experiments.

The fraction factorial design technique is widely used because the exponential disadvantage of the full factorial design is compensated by taking a fraction like $1 / 2,1 / 4$, etc. of the full factorial design. Such a technique is useful in the early stage of a project to define the main factors with the biggest impact. There are three main ideas on which this technique is based, [18]:

1) The system with several variables is driven by the main effects and low-order interactions.

2) It is possible to project the fractional factorial design into larger designs

3) It is possible to combine the runs of two (or more) fractional factorials to assemble sequentially a larger design.

The second main goal of this paper is an identification of the relation between the input data and the output data. Therefore, $2^{k}$ full factorial design approach with the software Design Expert is used on an illustrative example to identify the main effects and their interactions.

\subsection{The Illustrative Example}

The illustrative example is a plate prefabrication line, which is part of every shipyard. Such a line is built up by five machines, flattening, drying, blasting preserving and marking, Fig. 2. The machines are characterized by the operational probability $p_{i}$, where $i$ is the number of the machine in the line. The plates are moving on a conveyor from machine to machine. The space between the machines defines the buffer capacity, $N_{j}$, where $\mathrm{j}$ is the number of the buffer in the line. In this example, we assume that there will be just one dimension of plates, which are passing through the production line. The prefabrication line is defined by 5 variable operational probabilities $\left(p_{1}, p_{2}, p_{3}, p_{4}, p_{5}\right)$ and 4 variable buffer capacities $\left(N_{1}, N_{2}, N_{3}, N_{4}\right)$. These 9 factors generate 14 output key parameters, the production rate $P R$, the work in process $W I P_{1}, W I P_{2}, W I P_{3}, W I P_{4}, W I P$, the blockade $B L_{1}, B L_{2}, B L_{3}, B L_{4}$, and the starvation $S T_{2}, S T_{3}, S T_{4}$, $S T_{5}$.

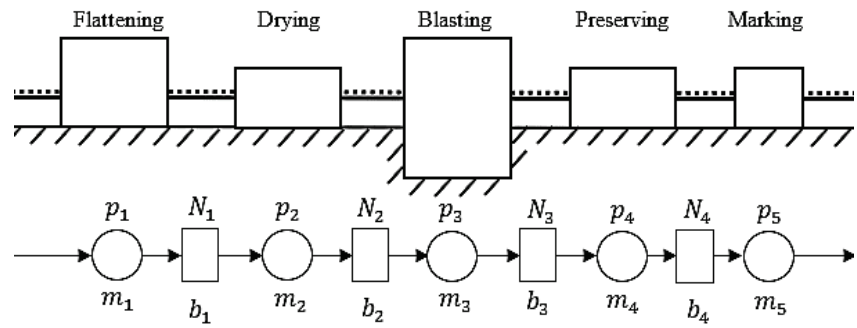

Figure 2 Plate prefabrication line and mathematical model, recreated according to [13]

The full factorial design of experiment is taken these 9 factors into consideration. Each factor has two levels, a high level and a low level, see Tab. 1. Therefore, $2^{9}=512$ experiments will be needed to discover all possible combinations.

\begin{tabular}{|} 
Table 1 Input data for the full factorial design experiment \\
\begin{tabular}{|c|c|c|}
\hline & $p_{1-5}$ & $N_{1-4}$ \\
\hline low level & 0.6 & 1 \\
\hline high level & 0.9 & 5 \\
\hline
\end{tabular}
\end{tabular}

To generate such an amount of experiments we decided to use the fast finite state method as the statistical comparison shows that it generates very similar key parameters within an Error-range of $1 \%$, according to the analytical approach.

\subsection{The Results}

The full factorial design of experiments is run by the software Design Experts. Analyzing each key parameter in correlation with the factors, we get plots that visualize the trend of changing. Fig. 3 illustrates the trend of changing of the key parameter production rate, $P R$, by changing each factor according to Tab. 1. The results of the largest two main effects are listed in Tab. 2. Each key parameter will on average increase or decrease depending by the largest two main effects. 

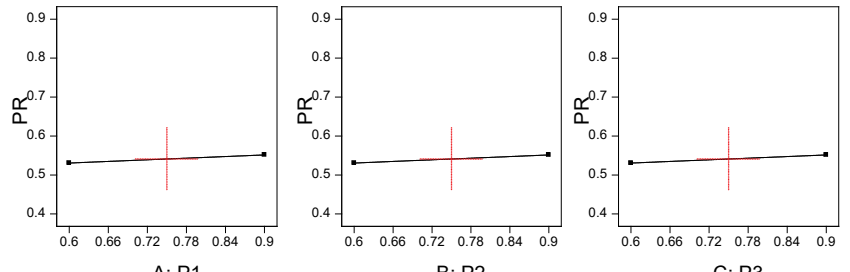

B: P2
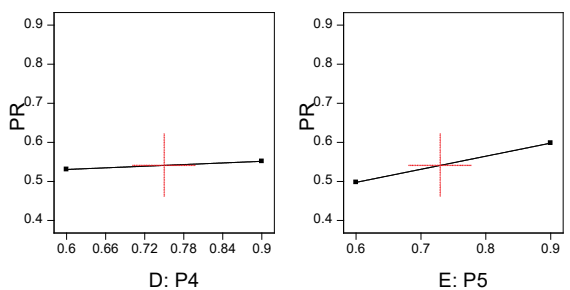

C: P3
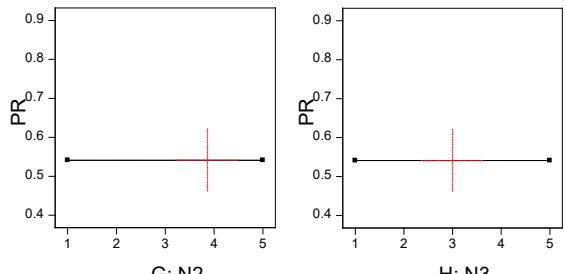

$\mathrm{H}: \mathrm{N} 3$

Figure 3 Effects related to the production rate
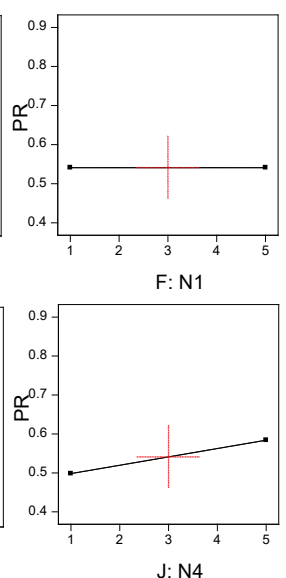

J: N4

Table 2 Largest main effects according to the output parameters

\begin{tabular}{|c|c|c|c|}
\hline Output key parameters & \multicolumn{3}{|c|}{ Largest main effects } \\
\hline \multirow{2}{*}{$P R$} & $N_{4}$ & 0.10 & \multirow{2}{*}{ pc/cycle time } \\
\hline & $p_{5}$ & 0.08 & \\
\hline \multirow{2}{*}{$W I P_{1}$} & $N_{1}$ & 2.44 & \multirow{2}{*}{$\mathrm{pc}$} \\
\hline & $p_{1}$ & 1.58 & \\
\hline \multirow{2}{*}{$W I P_{2}$} & $N_{2}$ & 1.74 & \multirow{2}{*}{ pc } \\
\hline & $p_{3}$ & -0.84 & \\
\hline \multirow{2}{*}{$W I P_{3}$} & $N_{3}$ & 1.40 & \multirow{2}{*}{$\mathrm{pc}$} \\
\hline & $p_{4}$ & -0.96 & \\
\hline \multirow{2}{*}{$\mathrm{WIP}_{4}$} & $N_{4}$ & 1.24 & \multirow{2}{*}{ pc } \\
\hline & $p_{5}$ & -1.02 & \\
\hline \multirow{2}{*}{$W I P$} & $N_{1}$ & 2.44 & \multirow{2}{*}{$\mathrm{pc}$} \\
\hline & $p_{1}$ & 2.84 & \\
\hline \multirow{2}{*}{$B L_{1}$} & $N_{1}$ & -0.08 & \multirow{2}{*}{ probability } \\
\hline & $p_{1}$ & 0.28 & \\
\hline \multirow{2}{*}{$B L_{2}$} & $N_{2}$ & -0.12 & \multirow{2}{*}{ probability } \\
\hline & $p_{2}$ & 0.15 & \\
\hline \multirow{2}{*}{$B L_{3}$} & $N_{3}$ & -0.12 & \multirow{2}{*}{ probability } \\
\hline & $p_{4}$ & -0.11 & \\
\hline \multirow{2}{*}{$B L_{4}$} & $N_{4}$ & -0.10 & \multirow{2}{*}{ probability } \\
\hline & $p_{5}$ & -0.12 & \\
\hline \multirow{2}{*}{$S T_{2}$} & $p_{1}$ & -0.19 & \multirow{2}{*}{ probability } \\
\hline & $p_{2}$ & 0.11 & \\
\hline \multirow{3}{*}{$S T_{3}$} & $p_{3}$ & 0.16 & \multirow{3}{*}{ probability } \\
\hline & $p_{2}$ & -0.09 & \\
\hline & $p_{1}$ & -0.09 & \\
\hline \multirow{2}{*}{$S T_{4}$} & $N_{3}$ & -0.08 & \multirow{2}{*}{ probability } \\
\hline & $p_{4}$ & 0.19 & \\
\hline \multirow{2}{*}{$S T_{5}$} & $N_{4}$ & -0.08 & \multirow{2}{*}{ probability } \\
\hline & $p_{5}$ & 0.20 & \\
\hline
\end{tabular}

The production rate mainly depends on the last buffer and machine in the line. When the buffer capacity $N_{4}$ is increasing from 1 to 5 , the production rate, $P R$, will increase on average 0.1 pieces per cycle time. The change of the operational probability of the fifth machine, $p_{5}$, from 0.6 to 0.9 will result that the $P R$ increase on average 0.08 pieces per cycle time. The work in process, $W I P_{j}$, depends on the capacity $N_{j}$ of the $j^{\text {th }}$ buffers in the production line. If the capacity increase on average from 1 to 5 the $W I P_{j}$ will increase between 1.4 pieces and 2.44 pieces. The work in process all, WIP, mainly depends on the buffer capacity $N_{4}$ and on the operational probability of the first machine, $p_{1}$. Among the probability of blockade factors, the $B L_{1}$ has the biggest main effect. When the operational probability of the first machine, $p_{1}$, is changing from 0.6 to 0.9 the key parameter $B L_{1}$ will on average increase by 0.28 . The probability of starvation of the third machine, $S T_{3}$, has three dominant main effects. The biggest change is caused by the increase of the operational probability of the fifth machine, $p_{5}$.

\section{CONCLUSION}

The statistical comparison shows that the numerical approach generates some higher values than the analytical approach, but the results are still good enough. The aggregation method and the finite state method are very accurate according to the analytical approach.

The design of experiment shows the interaction between the key parameters and the input parameters (factors). Analyzing the data, we can conclude that if we want to increase the production rate of the production line in the illustrative example, that increasing the buffer capacity $N_{4}$ and the operational probability of the fifth machine, $p_{5}$ will be the best choice. Further researches could be to run a Central Composite Face (CCF) centered design technique with three levels, which would better approximate the changing of the output key parameters. In this case, the user could change the factors within the range of the model and simulate different scenarios of the production line.

\section{Acknowledgment}

The research is supported by the Croatian Science Foundation, project UIP-2019-04-6573 ANTYARD (Advanced Methodologies for Cost Effective, Energy Efficient and Environmentally Friendly Ship Production Process Design).

\section{Notice}

The paper was presented at MOTSP $2021-12^{\text {th }}$ International Conference Management of Technology - Step to Sustainable Production, which took place in Poreč/ Porenzo, Istria (Croatia), on September 8-10, 2021. The paper will not be published anywhere else.

\section{REFERENCES}

[1] Kurfess, T. (2013). Why Manufacturing Matters. Mechanical Engineering, 135(11), 32-35. https://doi.org/10.1115/1.2013-NOV-1

[2] Bivens, J. (2019). Updated employment multipliers for the U.S. economy. Economic Policy Institute, Washington DC.

[3] Wang, Z. \& Gershwin, S. B. (2015) Heuristic production and sale policy for a two-product-type manufacturing system with 
downward substitution. IEEE Transactions on Systems, Man, and Cybernetics: Systems, 929-942. https://doi.org/10.1109/TSMC.2014.2371876

[4] Jia, Z., Zhang L., Arinez, J., \& Xiao, G. (2016). Performance analysis for serial production lines with Bernoulli Machines and Real-time WIP-based Machine switch-on/off control. International Journal of Production Research, 54(21), 62856301. https://doi.org/10.1080/00207543.2016.1197438

[5] Zhou, W., Wang, J., \& Zhu, X. (2019). Research on Environmental Assessment Model of Shipyard Workshop Based on Green Manufacturing. Journal of Coastal Research, 16-20. https://doi.org/10.2112/SI94-004.1

[6] Hadžić, N., Ložar, V., Opetuk, T., \& Andrić, J. (2021). A Finite State Method in improvement and design of lean Bernoulli serial production lines. Computers \& Industrial Engineering, 159, 107449. https://doi.org/10.1016/j.cie.2021.107449

[7] Sevast'yanov, B. A. (1962). Influence of Storage Bi Capacity on the Average Standstill Time of Production Line. Theory of Probability Applications, 429-438. https://doi.org/10.1137/1107040

[8] Li, J. \& Meerkov, S. M. (2009). Production System Engineering, Springer, New York. https://doi.org/10.1007/978-0-387-75579-3

[9] Gershwin, S. B. (1986). An efficient decomposition method for the approximate evaluation of tandem queues with finite storage space and blocking. Operation Research, 35(2), 291305. https://doi.org/10.1287/opre.35.2.291

[10] Dallery, Y., David, R., \& Xie, X. L. (1988). An efficient algorithm for analysis of transfer lines with unreliable machines and finite buffers. IIE Transactions, 20(3), 280-283, https://doi.org/10.1080/07408178808966181

[11] Dallery, Y. \& Bihan, H. L E. (1999). An improved decomposition method for the analysis of production lines with unreliable machines and finite buffers. International Journal of Production Research, 37:5, 1093-1117 https://doi.org/10.1080/002075499191427

[12] Lim, J.-T., Meerkov, S., \& Top, F. (1990). Homogeneous asymptotically reliable serial production lines: Theory and a case study. IEEE Trans. Autom. Control., 35(5), 524-534. https://doi.org/10.1109/9.53518

[13] Hadžić, N. (2019). Analytical solution of the serial Bernoulli production line steady-state performance and its application in the shipbuilding process. International Journal of Production Research 57(4), 1052-1065. https://doi.org/10.1080/00207543.2018.1500042

[14] Hadžić, N., Ložar, V., \& Abdulaj, F. (2020). A Finite State Method in the performance evaluation of the Bernoulli serial production lines. Applied sciences, 10(18), 6602. https://doi.org/10.3390/app10186602

[15] Václav, Š. \& Lecký, Š. (2017). Impact of computer-aided assembly technologies and simulation. International Scientific Journal "INDUSTRY 4.0", II(1), 9-12.

[16] Ložar, V., Hadžić, N., Opetuk, T., \& Slapničar, V. (2021). Accuracy evaluation of the semi-analytical and numerical methods in the production engineering, Mathematics, 9(13), 1461. https://doi.org/10.3390/math9131461

[17] Cowles, H. M. (2015). Hypothesis Bound: Trial and Error in the Nineteenth Century. Isis, 106(3), 635-645. https://doi.org/10.1086/683528

[18] Montgomery, D. C. (2001). Design and analysis of experiments. $5^{\text {th }}$ ed. New York: John Wiley.

\section{Authors' contacts:}

Viktor Ložar, univ. mag. ing. Naval Architect

(Corresponding author)

University of Zagreb,

Faculty of Mechanical Engineering and Naval Architecture, Ivana Lučića 5, 10000 Zagreb, Croatia

E-mail: viktor.lozar@fsb.hr

Tihomir Opetuk, PhD Assistant Professor

University of Zagreb,

Faculty of Mechanical Engineering and Naval Architecture, Ivana Lučića 5, 10000 Zagreb, Croatia

E-mail: thomir.opetuk@fsb.hr

Hrvoje Cajner, PhD Associate Professor

University of Zagreb,

Faculty of Mechanical Engineering and Naval Architecture, Ivana Lučića 5, 10000 Zagreb, Croatia

E-mail: hrvoje.cajner@fsb.hr

Neven Hadžić, PhD Associate Professor

University of Zagreb,

Faculty of Mechanical Engineering and Naval Architecture, Ivana Lučića 5, 10000 Zagreb, Croatia

E-mail: neven.hadzic@fsb.hr

Jerolim Andrić, PhD Full Professor

University of Zagreb,

Faculty of Mechanical Engineering and Naval Architecture, Ivana Lučića 5, 10000 Zagreb, Croatia

E-mail: jerolim.andric@fsb.hr 